\title{
MFC/NFC aerogel-Ag composite and its application
}

\author{
Jin HuaYan ${ }^{1, a^{*}}$, Ren Mei Xü,b \\ ${ }^{1}$ Guangdong Industry Technical College, China \\ ${ }^{2}$ Ball State University, USA \\ a Jhyan2000@yahoo.com, brxu@bsu.edu \\ * Corresponding author
}

Keywords: MFC/NFC; Aerogel; Nanoparticles; Antibacterial activity; Composite

\begin{abstract}
MFC/NFC aerogel was prepared, silver nanoparticles (AgNPs) were loaded in the aerogel. Ag-NPS were obtained successfully in the matrices without adding any other binders. The aerogel AgNPS size was defined by TEM. The thermal-stability was marked by TGA. This aerogel showed superior properties for Ag-NPS loading and excellent antibacterial activity. MFC/NFC aerogel will have potential application in antibacterial substrate.
\end{abstract}

\section{Introduction}

Metal particles in the nanometer size range have attracted considerable interest in recent years, as they have many attractive applications in various fields [1-6] due to their unique size-dependent optical, electrical, magnetic, and antimicrobial properties. Silver nanoparticles (AgNPs) are believed to be size-dependent to their antimicrobial properties, where smaller AgNPs provide stronger antimicrobial effect than larger AgNPs [7,8]. The size of the nanoparticle implies that it has a large surface area to come in contact with bacterial cells and hence, it will have a higher percentage of interaction than bigger particles [9-11]. In this case, aggregation of AgNPs must be avoided because it will drastically decrease the accessibility of nanoparticles' surfaces, resulting in insufficient functionality. It is basically difficult to disperse metallic nanoparticles in a solvent, as nanoparticles tend to aggregate due to their high surface energy. Facile synthesis of morphologically controlled nanoparticles is a significant challenge in the field of nanotechnology.

In this work, we provide a different method to load nanoparticles using MFC/NFC aerogel as templates. MFC/NFC aerogel was prepared, then nanopartilces was generated on the aerogel. Silver was an example. This biocompatible AgNPs/matrix composite was found to exhibit excellent anti-microbial activities for Escherichia coli. Generally, MFC-based AgNPs aerogel showed the best properties for nanoparticles loading than NFC aerogel.

\section{Experimental part.}

Silver NPs (AgNPs) loading. MFC/NFC aerogel was prepared in our lab. In situ loading of silver nanoparticles onto the aerogel was carried out through the reduction of $10 \mathrm{mM} \mathrm{AgNO}_{3}$ solution. Under ambient temperature $\left(\sim 25^{\circ} \mathrm{C}\right)$, several drops of $\mathrm{AgNO}_{3}$ solution were spread on the porous matrix, keeping totally wet for enough long time till the substrates were not absorbing solution. It is similar to that the substrates were soaked in $\mathrm{AgNO}_{3}$ solution for saturation and uniformity, but much less solution was used in this experiment.

After a certain time of air drying, a partially dehydrated matrix was obtained. It was then immersed in aqueous solution of $\mathrm{NaBH}_{4}(50 \mathrm{mM})$ for $20 \mathrm{~min}$. Meanwhile, the color of the resultant samples turned to yellow or dark brown due to the reduction of $\mathrm{Ag}^{+}$into silver nanoparticles. The composite was rinsed with Milli-Q water three times to remove water-soluble substances and free silver particles. Finally, the composite was freeze dried. 
TEM preparation of MFC/NFC aerogels with ANPs. A JEOL 2000FX transmission electron microscope (TEM) operating at $20.0 \mathrm{kV}$ was utilized to define the AgNPs in the MFC/NFC aerogels. A pecimen can be prepared by cutting the sample into thin slices using a diamond saw, then cutting 3-mm-diameter disks from the slice, thinning the disk on a grinding wheel, dimpling the thinned disk, then ion milling it to electron transparency.

Antibacterial activity test. The antibacterial activity of the aerogels were tested against Escherichia coli (E. coli) Gram negative bacteria, using the viable cell counting method. Briefly, about $100 \mu \mathrm{L}$ E. coli was cultivated in $100 \mathrm{~mL}$ of a nutrient broth solution, to give a bacterial concentration of about $7 \times 1011 \mathrm{CFU} / \mathrm{mL}$. Then, $1 \mathrm{~mL}$ of the bacteria/nutrient solution was added to 9 $\mathrm{mL}$ of sterilized nutrient broth solution $(0.8 \%)$. Several decimal dilutions were performed until the bacterial concentration increased from $7 \times 103$ to $7 \times 107 \mathrm{CFU} / \mathrm{mL}$. NFC, NFC/chitosan, MFC/chitosan, and their silver loaded counterparts, NFC/chitosan/Ag-NPs, MFC/chitosan/Ag-NPs, were used in the antibacterial tests. Accurate weight of the aerogel samples $(100 \mathrm{mg})$ was assigned to the experiment. To perform the antibacterial testing, aerogels of the respective samples were put into $10 \mathrm{~mL}$ of the bacteria/nutrient solution incubated in a shaker at $37{ }^{\circ} \mathrm{C}$ for $12 \mathrm{~h}$. After the exposure of the bacteria to films, $100 \mu \mathrm{L}$ of the bacterial solution was taken out and quickly spread on a plate containing nutrient agar. Plates containing bacteria were incubated at $37{ }^{\circ} \mathrm{C}$ for $24 \mathrm{~h}$, and then the numbers of the surviving colonies were counted. These results were compared to the number of bacteria colonies of the untreated control.

\section{Results and discussions}

Formation of AgNPs on MFC/NFC aerogel. The MFC aerogel and NFC aerogel microstructures were shown below in Figure.1. In Fig.1, we can see the difference of MFC aerogel and NFC aerogel. MFC aerogel has open structure and has more pores, the texture is more uniform. NFC aerogel seems tighter and has less pores. NFC holds more carboxyl groups and more easily to form hydrogen bonds. We can image that MFC aerogel will have good performance to nanoparticles loading and filling.
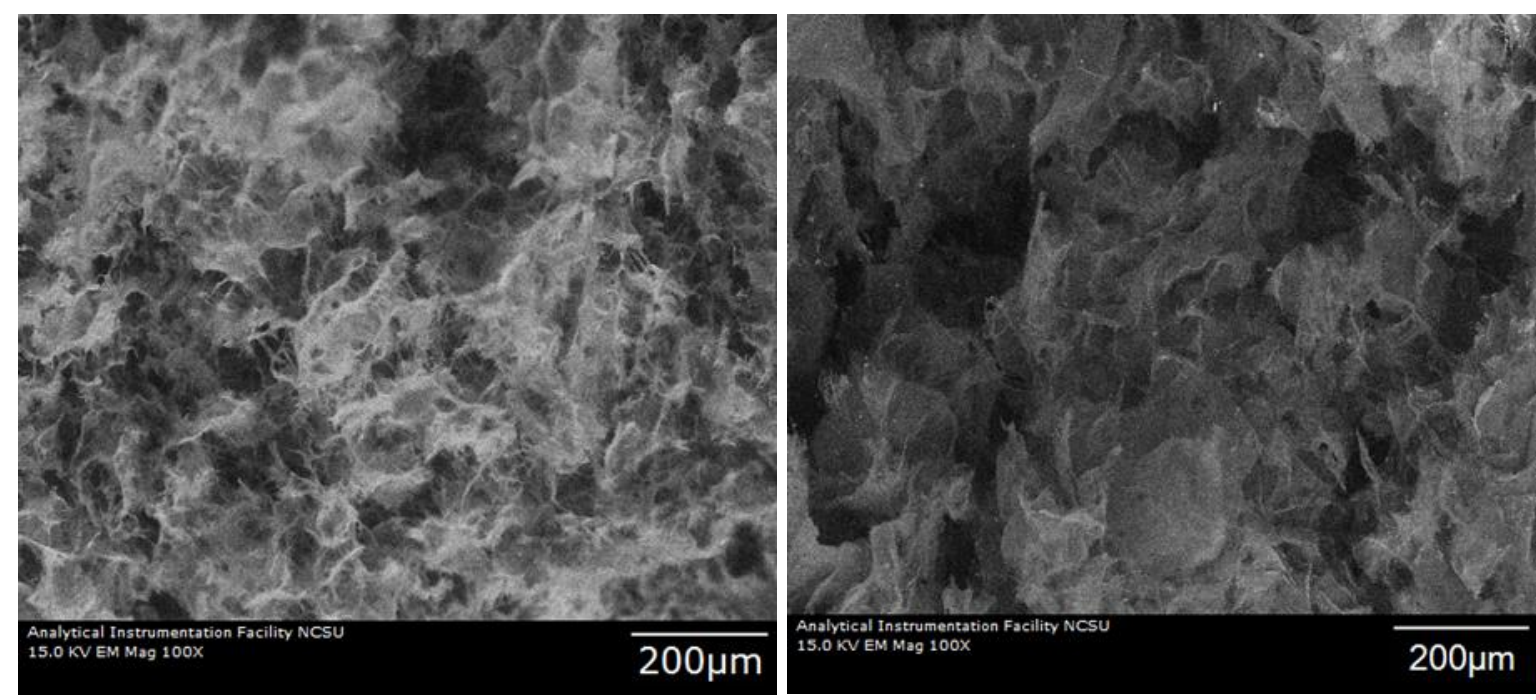

Figure.1 MFC aerogel (left) and NFC aerogel (right) SEM images

After AgNPs loading, the aerogels structure and nanoparticle distribution were shown in Figure.2. In Fig.2, MFC aerogel has better particles distribution than NFC aerogel, which the nanoparticles scattered more evenly in MFC aerogel. 

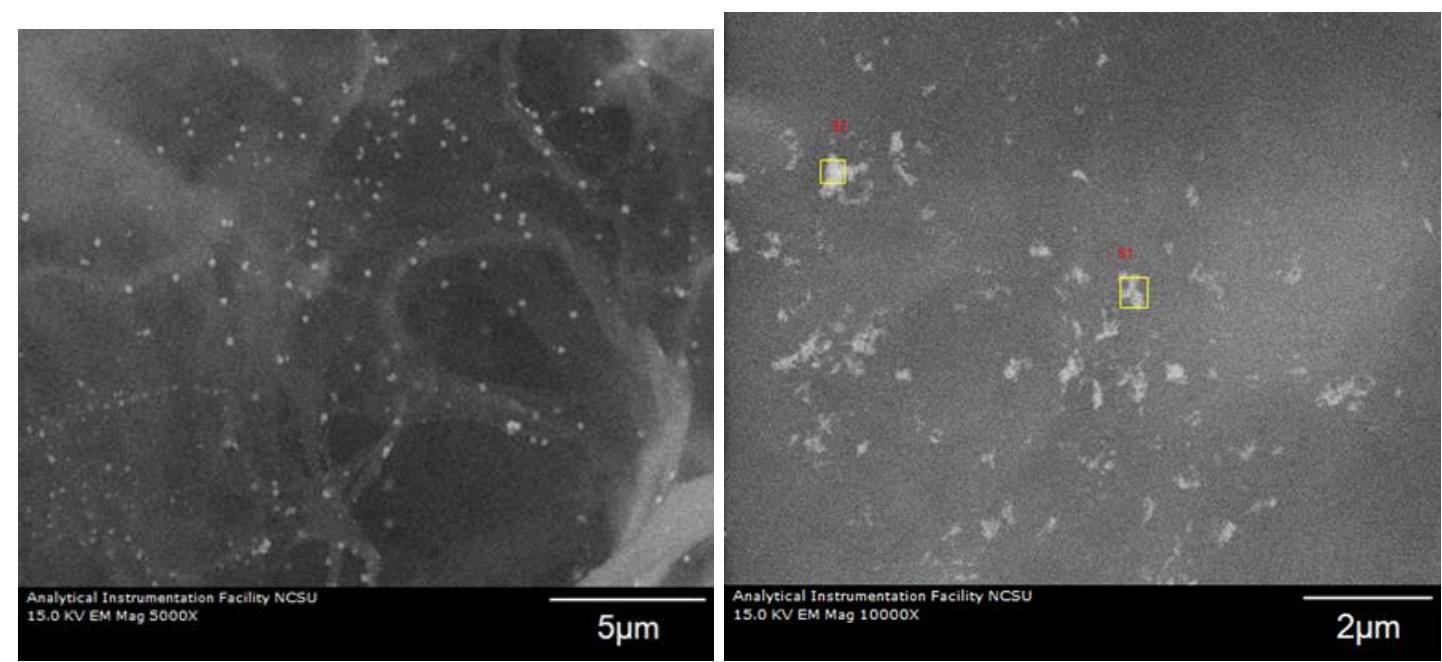

Figure. 2 MFC aerogel (left) and NFC aerogel (right) SEM images after AgNPs loading

In order further to know AgNPs size and distribution for MFC aerogel, TEM was conducted (see Figure.3). The particles are mono-dispersed and have very small size with mean diameter of $7.08 \mathrm{~nm}$, most particles in the range of 4-9nm. This is because the aerogel was treated by very dilute AgNO3 solution $(10 \mathrm{mM})$, there were less Ag+ surrounding nanofibrils, typically at negative charged groups which are the anchors to be nucleus of particle growth. Nanofibrils placed an important role to physically stop the Ag+ free move, then mono-dispersed small particles were formed.
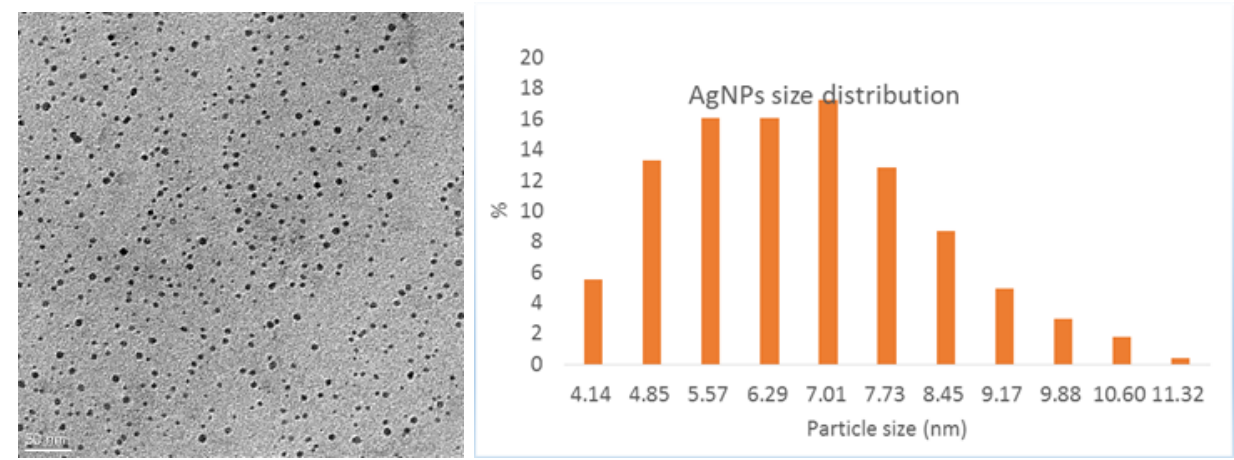

Figure. 3 TEM images of MFC aerogels AgNPs distribution

TGA test. In view of the importance of thermal stability in many applications of AgNPs loaded MFC/NFC aerogels, we examined thermal decomposition of composites loaded AgNPs by thermogravimetry (TGA) in a nitrogen atmosphere under $500^{\circ} \mathrm{C}$, as shown in Figure.4. In all TGA curves, the small weight losses below $150^{\circ} \mathrm{C}$ apparently resulted from evaporation of adsorbed moisture. Under nitrogen, the most weight loss of the MFC-Ag aerogel taking place at $280^{\circ} \mathrm{C}$, meanwhile, NFC-Ag aerogels have lower decomposition temperature of $250^{\circ} \mathrm{C}$ around. In order to obtain the AgNPs amount in aerogel, TGA under oxygen in the range of $500-575^{\circ} \mathrm{C}$ was continued. The isothermal time for $25 \mathrm{~min}$ at $575^{\circ} \mathrm{C}$ was set up. The AgNPs amount in MFC aerogel has more than that in NFC aerogel. 


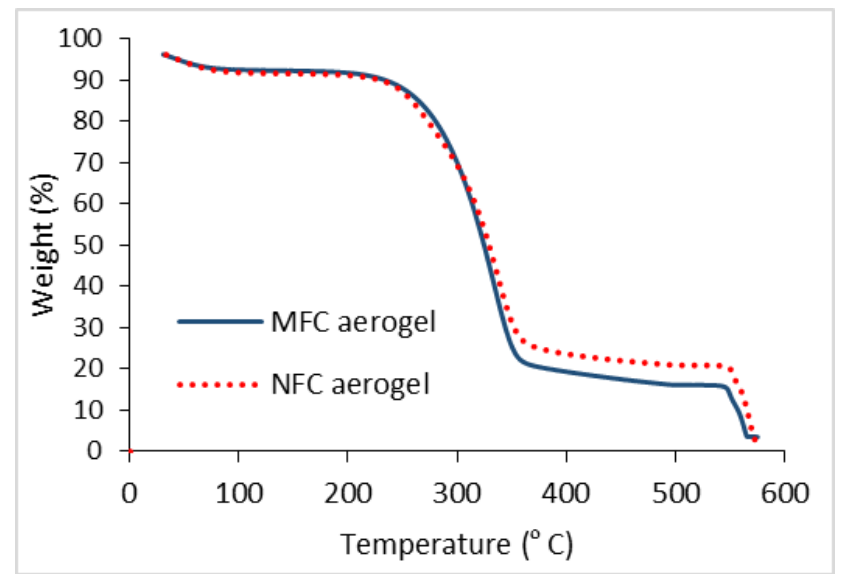

Figure. 4 MFC/NFC aerogels TGA

Antibacterial activity test. The antibacterial activity of MFC/NFC aerogels was tested against E. coli .by using the viable cell-counting method. The effects of aerogels on the growth of the recombinant bacteria E. coli are shown in Figure.5. As shown in the plates, no bacterial colonies was observed at concentrations of $7 \times 10^{7} \mathrm{CFU} / \mathrm{mL}$ for both MFC aerogel $-\mathrm{AgNPs}$ and NFC aerogel-AgNPs which represents the highest antibacterial activity. A lot of bacterial colonies were observed for MFC and NFC aerogels which implied the poor antibacterial activity. AgNPs in these aerogels imported great inhibition of bacterial. MFC aerogel -AgNPs may have better antibacterial property than NFC aerogel-AgNPs, since the former has excellent nanoparticles distribution. This should be further examined.

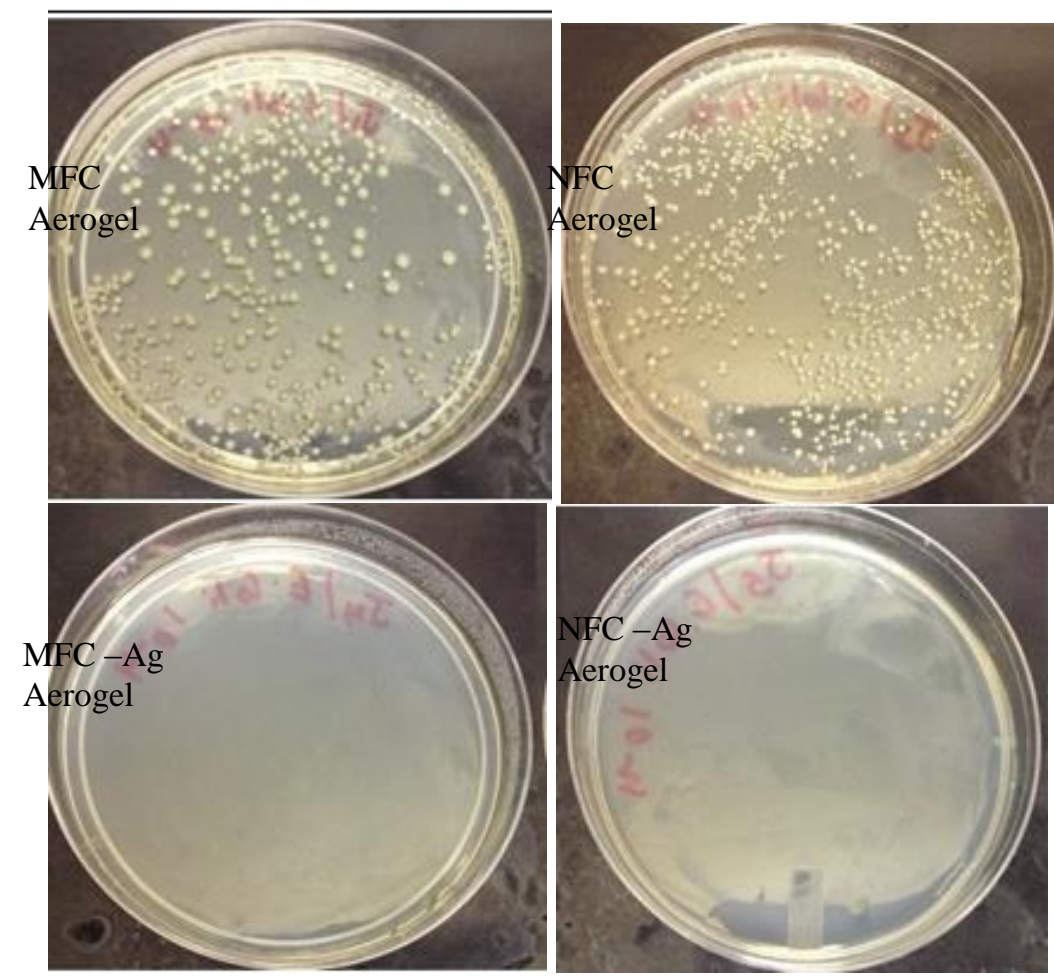

Figure. 5 Antibacterial activity of MFC aerogel, NFC aerogel, MFC-AgNPs aerogel, and NFC-AgNPs aerogel against $E$. coli.

\section{Conclusion}

In this work we have shown that MFC/NFC aerogel can be facilely used as substrate for preparation and stabilization of nanoparticles of AgNPs. AgNPs are well dispersed on the MFC aerogel. The MFC/NFC aerogel-AgNPs hybrid performed strong antibacterial properties. We therefore propose 
that the procedure described here, the metal ions are anchoring on the aerogel due to their negative charged groups, to first perform chemical reactions as the core, and then grow particles, will provide a technically more feasible procedure. The work is also a demonstration that highly fibrillated MFC/NFC aerogel shows desirable properties that lead to new methods in technology. MFC aerogel is better for AgNPs loading than NFC aerogel here.

\section{References}

[1] Z. Shervani, Y. Ikushima, M. Sato, H. Kawanami, Y. Hakuta, T. Yokoyama, T.Nagase, H. Kuneida, K. Aramaki, Colloid Polym. Sci. 286 (2008) 403.

[2] Zhong C and Maye M M, Core-Shell Assembled Nanoparticles as Catalysts, 2001 Adv. Mater. 13 1507

[3] Kim Y, Johnson R C and Hupp J T, Gold Nanoparticle-Based Sensing of 'Spectroscopically Silent" Heavy Metal Ions, 2001 Nano Lett. 1165

[4] J. Zhu, S. Liu, O. Palchik, Y. Koltypin, A. Gedanken, Shape-controlled synthesis of silver nanoparticles by pulse sonoelectrochemical methods, Langmuir 16 (2000) 6396.

[5] M. Li, H. Schnablegger, S. Mann, Coupled synthesis and self-assembly of nanoparticles to give structures with controlled organization, Nature 402 (1999) 393.

[6] C. Chen, L. Wang, H. Yu, J. Wang, J. Zhou, Q. Tan, L. Deng, Morphology-controlled synthesis of silver nanostructures via a seed catalysis process, Nanotechnology 18 (2007) 115612-115620 .

[7] C. Chen, L. Wang, H. Yu, J. Wang, J. Zhou, Q. Tan, L. Deng, Nanotechnology 18 (2007) 115612.

[8]Mahndra Rai, Alka Yadav, Aniket Gade, Silver nanoparticles as a new generation of antimicrogials, Biotechnology Advances 27(2009) 76-83

[9] Morones JR, Elechiguerra JL, Camacho A, Ramirez JT. The bactericidal effect of silver nanoparticles. Nanotechnology 2005; 16:2346-53

[10] Raimondi F, Scherer GG, Kotz R, Wokaun A. Nanoparticles in energy technology: examples from electochemistry and catalysis. Angew. Chem., Int. Ed. 2005; 44:2190-209.

[11] Pal S, Tak YK, Song JM. Does the antibacterial activity of silver nanoparticles depend on the shape of the nanoparticle? A study of the gram-negative bacterium Escherichia coli. Appl Environ Microbiol 2007; 27(6):1712-20. 